\title{
Conducta de ayuda en ratas: ¿acción o hábito?*
}

\author{
Help Behaviors in Rats: Action or Habit?
}

Recepción: 13 Febrero 2017 | Aceptación: 09 Noviembre 2018

\author{
RODOlFo Bernal-GambOA ${ }^{\mathrm{a}}$ \\ Universidad Nacional Autónoma de México, México \\ ORCID: http://orcid.org/0000-0002-4096-8425
}

a Autor de correspondencia. Correo electrónico: rbernalg@unam.mx

Para citar este artículo: Bernal-Gamboa, R. (2019). Conducta de ayuda en ratas: iacción o hábito? Universitas Psychologica, 18(1), 1-7. https://doi.org/1 0.11144/Javeriana.upsy18-1.cara

\section{RESUMEN}

En los últimos años, se han reportado varios estudios sobre la empatía en roedores. En particular, las tareas que evalúan los comportamientos de ayuda en ratas han propuesto que el mecanismo que subyace a dichas conductas es la empatía. Aunque varios autores apoyan dicha propuesta, existe evidencia inconsistente con el mecanismo empático. Por ejemplo, si se asume que la conducta de ayuda es motivada por la empatía, dicha conducta únicamente debería ejecutarse cuando sea necesaria (eliminar el distrés en otra rata). Sin embargo, recientemente se ha reportado que las ratas continúan realizando la conducta de ayuda incluso en situaciones en que dicho comportamiento no funciona. Se diseñó un experimento con ratas para evaluar si la cantidad de entrenamiento afecta la sensibilidad de la conducta de ayuda a la consecuencia (reducción del distrés). Se utilizó una tarea que implicó que una rata liberase a otra de un contenedor tubular. El Grupo Corto realizó dicha conducta durante pocas sesiones, mientras que el grupo Extenso ejecutó la conducta de ayuda por un periodo más largo. Finalmente, ambos grupos recibieron una prueba de generalización en la que el contenedor estuvo vacío. Los resultados mostraron que en las sesiones de prueba las ratas del Grupo Corto no abrieron el contenedor, mientras que el grupo Extenso sí lo abrió. Se discute el papel de los hábitos en la ejecución de conductas pro-sociales. Palabras clave acciones; conducta de ayuda; empatía; hábitos; ratas.

\begin{abstract}
In recent years, several authors have used rodent models to study empathy. In particular, it has been suggested that helping behavior in rats is motivated by empathic reasons. Although several authors support such perspective, some researchers have pointed out to inconsistent data. For example, if the mechanism underlying helping behavior is empathy, rats would perform that behavior only to end the distress in another rat. However, it has been observed that rats perform helping behavior even when that behavior does not work. One experiment with rats was conducted to explore whether the amount of training impairs the goaldirect component of the helping behavior. An experimental task that involved a free rat releasing a trapped rat in a restrainer was used. Group Short learned to open the restrainer for few sessions, whereas rats in Group Long opened the restrainer for an extensive period. Finally, both groups received a generalization test with an empty restrainer. Rats in Group Short did not open the restrainer during testing, while rats in Group Long did it. The role of habits in pro-social behaviors is discussed.
\end{abstract}


Keywords

actions; helping behavior; empathy; habits; rats.

Se ha propuesto que la respuesta al porqué los humanos somos tan amables con otros (incluso con desconocidos) puede tener raíces evolutivas (p. ej., Decety, Norma, Bernston, \& Cacioppo, 2012; Meyza, Ben-Ami Bartal, Monfils, Pankseep, \& Knapska, 2017). Por ello, una de las líneas de investigación que en años recientes ha recibido bastante atención por parte de los investigadores es el estudio de la prosocialidad en animales no humanos (MarshallPescini, Dale, Quervel-Chaumette, \& Range, 2016). La conducta pro-social es una conducta voluntaria emitida para brindar beneficio a otro (Cronin, 2012). Algunos autores han sugerido que el mecanismo que subyace a la pro-socialidad es la empatía (p. ej., de Waal, 2013).

Dado que la empatía involucra la comunicación de estados afectivos que (bajo las condiciones adecuadas) permiten la ejecución de comportamientos que reduzcan malestar en otros (Decety, 2011; Mason, 2014), varios investigadores han señalado la importancia de utilizar tareas que impliquen altruismo o conductas de ayuda (comportamiento voluntario para beneficio de otros pero que tiene un costo para el ejecutante). Así, utilizando primates no humanos, se ha reportado la habilidad de auxiliar a otro sujeto para que solucione un problema (p. ej., de Waal, 2008; Warneken \& Tomasello, 2006) o para que consiga un objeto o alimento (p. ej., Drayton \& Santos, 2014; Warneken, Hare, Melis, Hanus, \& Tomasello, 2007; Yamamoto, Humle, \& Tanaka, 2009).

Las conductas de ayuda también se han reportado en mamíferos no primates como las ratas (p. ej., Rice \& Gainer, 1962; Sato, Tan, Tate, \& Okada, 2015). En 2011, BenAmi Bartal, Decety y Mason publicaron una serie de experimentos que mostraron evidencia experimental de conductas de rescate en ratas. Dichos autores emplearon una tarea novedosa en la cual colocaron un par de ratas dentro de una caja de acrílico (arena). Una de las ratas (atrapada) se encerró en un contenedor tubular de acrílico transparente que se colocó en el centro de la arena. El contenedor tenía una puerta frontal que la rata atrapada no podía abrir, es decir, únicamente podía abrirse desde afuera. La otra rata (libre) podía moverse libremente por toda la arena. El estudio reportó la conducta de apertura de puerta por parte de la rata libre. Aunque dicha conducta no fue entrenada, las ratas la ejecutaron cada vez más rápido conforme avanzaron las sesiones. Los investigadores propusieron a la empatía como el mecanismo subyacente a la conducta de ayuda observada en las ratas. Es decir, sugirieron que la rata libre abrió la puerta de forma voluntaria para eliminar el distrés o malestar (provocado por la restricción de movimiento) en la rata atrapada (véanse también, Ben-Ami Bartal, Rodgers, Bernardez Sarria, Decety, \& Mason, 2014).

Aunque la explicación de Ben-Ami Bartal et al. (2011) ha recibido la aceptación de varios investigadores (p. ej., Bernal-Gamboa, 2017; de Waal, 2013; Mason, 2014; Mogil, 2012), el consenso no es general (Vasconcelos, Hollis, Nowbahari, \& Kacelnik, 2012). Por ejemplo, Silberberg, Allouch, Sandfort, Kearns, Karpel y Slotnik (2014), condujeron un estudio con ratas para evaluar si la empatía es el mecanismo subyacente a los comportamientos de ayuda en ratas. Empleando una tarea muy similar a la reportada por Ben-Ami Bartal et al. (2011; las ratas libres tocaban un sensor para permitir que las atrapadas salieran del contenedor tubular), diseñaron un experimento en el cual las respuestas al sensor ya no producían la liberación de la rata atrapada (Experimento 2). Silberberg et al. hipotetizaron que, si la conducta de ayuda estuviera motivada empáticamente, entonces dicha conducta debería extinguirse. Los autores reportaron que, sin importar que las respuestas al sensor no liberaran a las ratas atrapadas, las ratas libres continuaron ejecutando dicha conducta.

Silberberg et al. (2014) señalan que la persistencia de las ratas libres en ejecutar una conducta sin consecuencias sugiere incompatibilidad con el mecanismo empático, ya que si la conducta de ayuda (contacto con 
el sensor) estuviera reforzada por la liberación de la rata atrapada, las ratas libres deberían dejar de emitir dicha respuesta. Sin embargo, es importante notar que, en el experimento de Silberberg et al., las ratas recibieron casi cinco veces más exposición a la tarea (57 sesiones, mientras que Ben-Ami Bartal et al., en 2011, usaron 12). Lo anterior pudo favorecer que la conducta de ayuda fuera insensible a sus consecuencias, es decir que la persistencia de las ratas libres en ejecutar las respuestas al sensor (cuando ya no produjeron la liberación de la rata atrapada) pudiera demostrar el establecimiento de un hábito (Dickinson, 1985, 2011).

Por lo tanto, el objetivo del presente experimento fue evaluar lo anterior, a través del análisis de si la cantidad de exposición a la tarea experimental afecta la sensibilidad al estado actual de la consecuencia, en la conducta de ayuda en ratas. Para ello, dos grupos de parejas de ratas se expusieron a la tarea desarrollada por Ben-Ami Bartal et al. (2011). Uno de los grupos (Grupo Corto) recibió una exposición breve a la tarea (5 sesiones), mientras que el otro grupo de ratas (Grupo Extenso) fue expuesto a un mayor número de sesiones (15). Finalmente, ambos grupos recibieron una prueba de generalización sin la rata atrapada.

\section{Método}

\section{Sujetos}

Se usaron 16 parejas hembras de ratas Wistar experimentalmente ingenuas, de aproximadamente tres meses de edad al inicio del experimento. Las ratas se alojaron en parejas en cajas de metracrilato $(21 \times 24 \times 46 \mathrm{~cm})$, dentro de una habitación en un ciclo de luzoscuridad de 12-12 (las luces se prendían a las 7:00 y se apagaban a las 19:00). Todas las ratas se marcaron diariamente en la cola con un plumón de diferente color. Las ratas se manipularon diariamente dos semanas antes del inicio del experimento. La temperatura de la habitación donde se mantuvieron las ratas, se mantuvo entre 20 y $25^{\circ} \mathrm{C}$, mientras que la humedad se mantuvo entre el 45 y el $60 \%$. Todas las ratas se mantuvieron con acceso libre a comida y agua durante todo el experimento.

\section{Instrumentos}

Se emplearon instrumentos similares a los utilizados por Ben-Ami Bartal et al. (2011). Así, se usó una arena de acrílico transparente $(50 \mathrm{x}$ $50 \mathrm{~cm}$ ). Al centro de dicha arena se colocó un contenedor para rata $(25 \times 8.75 \times 7.5 \mathrm{~cm})$, el cual se mantuvo fijo durante toda la sesión. El contenedor tenía orificios en uno de los extremos, los cuales permitieron contacto físico y olfatorio entre las ratas. En el otro extremo, se colocó una puerta de dos paneles de acrílico transparente, unidos por tres tornillos y una polea $(5 \mathrm{~cm})$ en la que se colocaron dos pesas de $25 \mathrm{~g}$ para favorecer la caída de la puerta una vez que la rata la levantara. La rata libre podía levantar la puerta con la parte superior de su nariz o con su cabeza.

\section{Procedimiento}

El presente protocolo experimental fue aprobado por el Comité Ético de la Facultad de Psicología de la Universidad Nacional Autónoma de México. Todas las sesiones experimentales se condujeron en la misma franja horaria y en días consecutivos. Antes de empezar el experimento, se eligió al azar en cada pareja a la rata atrapada y a la rata libre. Las ratas no se mezclaron, es decir, la misma pareja se usó a lo largo de todo el experimento. Se utilizaron cinco días de pre-exposición. En el Día 1, se transportó a la habitación experimental a las ratas en parejas en sus cajas-hogar. Las ratas permanecieron en dicha habitación durante 40 minutos. Durante el Día 2 se realizó algo similar al día anterior, con la diferencia de que se manipuló gentilmente a cada una de las ratas durante 10 minutos. En los Días 3 a 5, se manipuló a cada rata durante cinco minutos y se colocó a ambas ratas (sin el contenedor) dentro de la arena durante 30 minutos. Después de cada sesión de preexposición, se regresó a las ratas al bioterio. 
Fase de Apertura

Al inicio de cada sesión, se introdujo a la rata atrapada dentro del contenedor tubular, se cerró la puerta y se colocó el contenedor al centro de la arena. Después se introdujo a la rata libre en la arena. Si la rata libre no abría la puerta del contenedor dentro de los primeros 40 minutos, el experimentador abría la puerta a la mitad, a un ángulo de $45^{\circ}$ para facilitar la apertura de la puerta. Después de cada apertura de puerta, las ratas permanecieron dentro de la arena los minutos restantes, para conformar una hora de sesión experimental. Se realizó una sesión diaria de esta fase para todas las ratas. Terminando cada sesión, se limpiaron la arena y el contenedor con una solución al 70 \% de alcohol. El Grupo Corto fue expuesto a 5 días de esta fase, mientras que las ratas en el Grupo Extenso recibieron tres veces más sesiones (15).

\section{Fase de Prueba}

Se condujeron tres sesiones de 40 minutos en circunstancias similares a las mencionadas en la fase anterior, Con la excepción de que el contenedor tubular estuvo vacío y únicamente la rata libre se colocó dentro de la arena. Se acomodaron las sesiones para que ambos grupos recibieran la prueba en los mismos días.

\section{Análisis Estadísticos}

Los promedios de latencias de aperturas de puerta fueron comparados a través de un ANOVA. Para comparar el porcentaje de aperturas de puerta entre los grupos se utilizó una prueba $t$. En todos los casos el criterio de rechazo establecido fue $p$ $<0.05$.

\section{Resultados}

En el lado izquierdo de la Figura 1, se muestra el promedio del número de aperturas de puerta de ambos grupos durante la Fase de Apertura. En dicha figura, se puede observar que conforme avanzan las sesiones los Grupos Corto y Extenso, abren más veces la puerta $(t(14)=8.56, p$ $=0.002$ ). Adicionalmente, el análisis estadístico confirmó que ambos grupos mostraron una ejecución similar en las primeras cinco sesiones de la Fase de Apertura $(t(14)=0.18, p=0.86)$.

En el lado derecho de la Figura 1, se muestra la media del número de aperturas de puerta durante la Fase de Prueba para los Grupos Corto y Extenso. El análisis estadístico confirmó que las ratas del Grupo Extenso abrieron significativamente más veces la puerta que el Grupo Corto $(t(14)=4.4, p=0.001)$.

\section{Figura 1}

Porcentaje promedio de aperturas de puerta durante las Fase de Apertura y Prueba de los Grupos Corto $y$ Extenso.

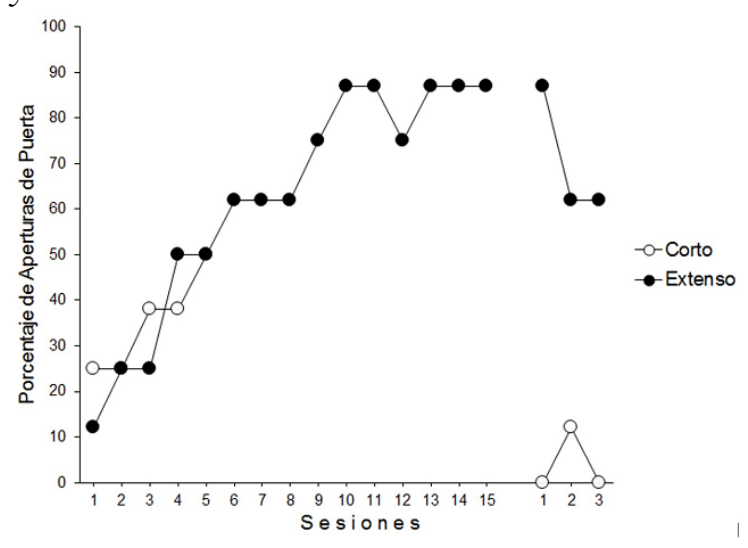

El promedio de latencias de aperturas de puerta durante la Fase de Apertura se muestra en el lado izquierdo de la Figura 2. Las ratas de los Grupos Corto y Extenso se comportaron de forma similar en dicha fase. Un ANOVA 2 (Grupo) x 5 (Sesión) conducido con las latencias de la Fase de Apertura confirmó que ambos grupos abrieron la puerta de forma más rápida conforme transcurrieron las sesiones. El ANOVA mostró un efecto significativo del factor Sesión, F $(4,56)$ $=6.71, p=0.001$. Ni el efecto principal Grupo, $F_{(1,14)}=0.15, p=0.71$, ni la interacción Grupo $\mathrm{x}$ Sesión $F_{(4,56)}=0.16, p=0.96$, resultaron significativos.

En el lado derecho de la Figura 2, se observa la media de latencias de aperturas de puerta a lo largo de la Fase de Prueba. Un ANOVA 2 (Grupo) x 3 (Sesión) conducido con las latencias 
de la Fase de Prueba confirmó que las ratas del Grupo Extenso abrieron más rápidamente la puerta durante estas sesiones. Dicho análisis mostró un efecto significativo del factor Grupo, $F_{(1,14)}=22.99, p=0.001$. El efecto principal Sesión, $F(2,28)=3.43, p=0.04$ y la interacción Grupo x Sesión $F_{(2,28)}=3.87, p=0.03$, resultaron significativos. Análisis subsecuentes mostraron que el Grupo Extenso abrió más rápido la puerta, $F_{(1,14)}=7.51, p=0.01$. Las comparaciones planeadas revelaron que las ratas en el Grupo Extenso tardaron menos en abrir la puerta en la sesión 1, $t(14)=48.59, p=0.001$, en la sesión 2, $t(14)=10.35, p=0.006$ y en la sesión $3, t(14)=11.33, p=0.004$.

\section{Figura 2}

Latencias promedio de aperturas de puerta de ambos grupos

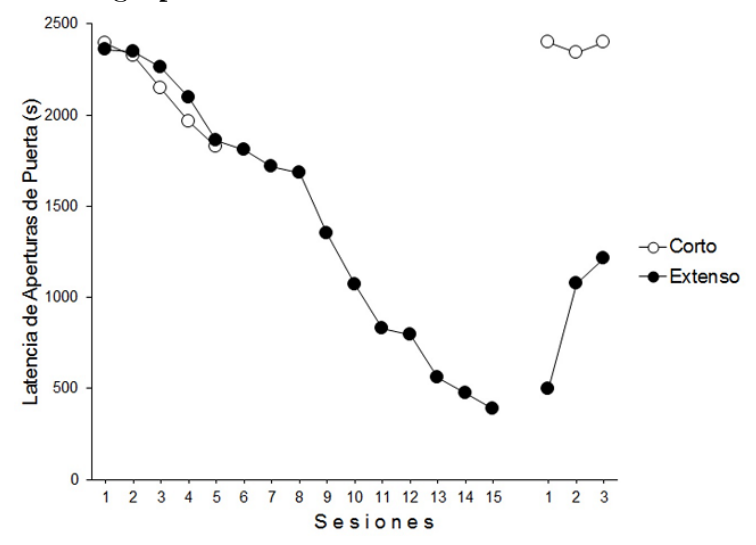

\section{Discusión}

El estudio aquí reportado se condujo con el objetivo de analizar si la conducta de rescate en ratas estaba motivada por razones empáticas. Para lo cual se condujo una fase que evaluara si la persistencia de dicha conducta en ausencia de consecuencia respondía a la formación de un hábito. Los datos mostraron que al inicio de la Fase de Apertura la conducta de ayuda es sensible a su consecuencia (i. e., es una acción dirigida a una meta), mientras que un mayor entrenamiento produce que el comportamiento altruista se convierta en un hábito (i. e., conducta insensible a la consecuencia).
Los resultados del presente experimento replican los hallazgos reportados por otros autores (p. ej., Ben-Ami Bartal et al., 2011, 2014; Silberberg et al., 2014) y demuestran que el presente paradigma experimental es válido para usarse en el estudio de conductas de ayuda en ratas. También es importante notar que la presente tarea resalta la generalidad de los comportamientos altruistas en ratas, dado que dichos comportamientos se han reportado usando diferentes cepas (Wistar, Long Evans, Sprague-Dawley).

De acuerdo con Silberberg et al. (2014), los hallazgos (la no extinción de la conducta de ayuda) sugieren que el mecanismo que subyace a la apertura de puerta no es empático. Sin embargo, los presentes resultados muestran que los resultados de dichos autores no descartan la participación de la empatía, sino que pueden explicarse debido al establecimiento de un hábito. Adicionalmente, el presente experimento señala que al igual que otras conductas instrumentales (i. e., dirigidas a una meta), los comportamientos altruistas son sensibles a la cantidad de entrenamiento.

Un mayor número de aperturas de puerta y una menor latencia en las mismas mostradas por el Grupo Extenso en la Fase de Prueba sugieren que la conducta de rescate se ha vuelto un hábito que es controlado por una asociación estímulo-respuesta ([E-R]; Hall, 2002). Por otro lado, la ejecución del Grupo Corto en la Fase de Prueba puede entenderse como una acción voluntaria que refleja el conocimiento de una asociación respuesta-consecuencia ([R-C]; Dickinson, 2011). Así, el presente experimento muestra que la conducta altruista puede ser controlada por procesos separados de acción y hábito.

Los presentes hallazgos se unen a otros que han demostrado distintas habilidades sociales en roedores motivadas por la empatía, tales como el contagio emocional (p. ej., Church, 1959; Langford et al., 2006, 2010) y la conducta prosocial (Hernández-Lallement, van Wingerden, Marx, Srejic, \& Kalenscher, 2015; Márquez, Rennie, Costa, \& Moita, 2015). Asimismo, es importante mencionar que, a diferencia de otras 
tareas experimentales empleadas en primates no humanos, el modelo desarrollado por BenAmi Bartal et al. (2011) ha resultado ser un paradigma robusto para estudiar la conducta de ayuda en animales no humanos, lo cual podría favorecer el estudio de los mecanismos evolutivos, cognitivos y neurobiológicos de los comportamientos altruistas.

\section{Agradecimientos}

El presente artículo contó con el apoyo del Proyecto PAPIIT IA302916.

\section{Referencias}

Ben-Ami Bartal, I., Decety, J., \& Mason, P. (2011). Empathy and pro-social behavior in rats. Sciene, 344, 1427-1430. https://doi.org /10.1126/science. 1210789

Ben-Ami Bartal, I., Rodgers, D. A., Bernardez, Sarria, M. S., Decety, J., \& Mason, P. (2014). Pro-social behavior in rats is modulated by social experience. eLife, 3, e01385. https:// doi.org/10.7554/eLife.01385.001s

Bernal-Gamboa, R. (2017). Conducta pro-social en ratas. Revista Argentina de Ciencias del Comportamiento, 9(1), 74-80. Recuperado de http://www.redalyc.org/pdf/3334/33345 0394009.pdf

Church, R. M. (1959). Emotional reactions of rats to the pain of others. Journal of Comparative and Physiological Psychology, 52, 132-134. https://doi.org/10.1037/h0043 531

Cronin, K. A. (2012). Prosocial behavior in animals: The influence of social relationships, communication and rewards. Animal Behaviour, 84, 1085-1093. https://d oi.org/10.1016/j.anbehav.2012.08.009

Decety, J. (2011). The neuroevolution of empathy. Annals of the New York Academy of Sciences, 1231, 35-45. https://doi.org/10.11 11/j.1749-6632.2011.06027.x

Decety, J., Norma, G. J., Bernston, G. G., \& Cacioppo, J. T. (2012). A neurobehavioral evolutionary perspective on the mechanisms underlying empathy. Progress in Neurobiology, 98, 38-48. https:// doi.org/10.1016/j.pneurobio.2012.05.001

de Waal, F. B. (2008). Putting the altruism back into altruism: The evolution of empathy. Annual Reviews of Psychology, 59, 279-300. https://doi.org/10.1146/annurev. psych.59.103006.093625

de Waal, F. B. (2013). The bonobo and the atheist. In search of humanism among the primates. Nueva York: W. W. Norton \& Company.

Dickinson, A. (1985). Actions and habits: The development of behavioural autonomy. Philosophical Transactions of The Royal Society of London Series B, Biological Sciences, 308, 67-78. https://doi.org/10.1098/rstb.19 85.0010

Dickinson, A. (2011). Goal-directed behavior and future planning in animals. En Menzel, R. \& Fischer, J. (Eds.), Animal thinking: Contemporary issues in comparative cognition (pp. 79-91). Cambridge, MA: MIT Press.

Drayton, L. A., \& Santos, L. R. (2014). Capuchins' (Cebus apella) sensitivity to others' goal-directed actions in a helping context. Animal Cognition, 17, 589-700. htt ps://doi.org/10.1007/s10071-013-0700-5

Hall, G. (2002). Associative structures in Pavlovian and instrumental conditioning. En C. R. Gallistel (Ed.), Stevens' handbook of experimental psychology (3.a ed., Vol. 3, pp. 1-45). Nueva York: John Wiley \& Sons.

Hernández-Lallement, J., van Windergen, M., Marx, C., Srejic, M., \& Kalenscher, T. (2015). Rats prefer mutual rewards in a prosocial choice task. Frontiers in Neuroscience, 8, 1-9. https://doi.org/10.338 9/fnins.2014.00443

Langford, D. J., Bailey, A. L., Chanda, M. L., Clarke, S. E., Drummond, T. E., Echols, S., ... Mogil, J. S. (2010). Coding of facial expressions of pain in the laboratory mouse. Nature Methods, 7, 447-449. https://doi.org /10.1038/nmeth.1455

Langford, D. J., Crager, S. E., Shehzad, Z., Smith, S. B., Sotocinal, S. G., Levenstadt, J. S., ... Mogil, J. S. (2006). Social modulation of pain as evidence for empathy in mice. 
Science, 312, 1967-1970. https://doi.org/10. 1126/science. 1128322

Marshall-Pescini, S., Dale, R., QuervelChaumette, M., \& Range, F. (2016). Critical issues in experimental studies of prosociality in non-human species. Animal Cognition, 19, 679-705. https://doi.org/10.1 007/s10071-016-0973-6

Márquez, C., Rennie, S. M., Costa, D. F., \& Moita, M. A. (2015). Prosocial choice in rats depends on food-seeking behavior displayed by recipients. Current Biology, 25, 1736-1745. https://doi.org/10.1016/j.cub.2 015.05.018

Mason, P. (2014). With a little help from our friends: How the brain processes empathy, Cerebrum, 14, 1-6. Recuperado de https://www.ncbi.nlm.nih.gov/pmc/artic les/PMC4445583/

Meyza, K. Z., Ben-Ami Bartal, I., Monfils, M. H., Pankseep, J. B., \& Knapska, E. (2017). The roots of empathy: Through the lens of rodent models. Neuroscience $\mathbb{E}$ Biobehavioral Reviews, 76, 216-234. https:// doi.org/10.1016/j.neubiorev.2016.10.028

Mogil, J. S. (2012). The surprising empathic abilities of rodents. Trends in Cognitive Sciences, 16, 143-144. https://doi.org/10.10 16/j.tics.2011.12.012

Rice, G. R., \& Gainer, P. (1962). "Altruism" in the albino rat. Journal of Comparative and Physiological Psychology, 55, 123-125. https: //doi.org/10.1037/h0042276

Sato, N., Tan, L., Tate, K., \& Okada, M. (2015). Rats demonstrate helping behavior toward a soaked conspecific. Animal Cognition, 18, 1039-1047. https://doi.org/10.1007/s10071 $-015-0872-2$

Silberberg, A., Allouch, C., Sandfort, S., Kearns, D., Karpel, H., \& Slotnik, B. (2014). Desire for social contact, not empathy, may explain "rescue" behavior in rats. Animal Cognition, 17, 609-618. https://doi.org/10.1007/s1007 1-013-0692-1

Vasconcelos, M., Hollis, K., Nowbahari, E., \& Kacelnik, A. (2012). Pro-sociality without empathy. Biology Letters, 8, 910-912. https:/ /doi.org/10.1098/rsbl.2012.0554
Warneken, F., Hare, B., Melis, A. P., Hanus, D., \& Tomasello, M. (2007). Spontaneous altruism by chimpanzees and young children. PLoS Biology, 5, 1414-1420. https ://doi.org/10.1371/journal.pbio.0050184

Warneken, F., \& Tomasello, M. (2006). Altruistic helping in human infants and young chimpanzees. Science, 311, 1301-1303. http s://doi.org/10.1126/science.1121448

Yamamoto, S., Humle, T., \& Tanaka, M. (2009). Chimpanzees help each other upon request. Plos One, 4, e 7416. https://doi.org/10.1371/ journal.pone. 0007416

\section{Notas}

* Artículo de investigación. 